\title{
Visualization of Ultrasound Propagation in the Glass with a Crack Possessing Residual Inner Stress
}

\author{
Kazuhiko IMANO and Yoshito HOSAKA* \\ * Graduate school of Engineering and Resource Science, Akita University \\ E-mail :imano@gipc.akita-u.ac.jp
}

\begin{abstract}
Visualization of $1 \mathrm{MHz}$ ultrasonic burst pulse propagation around a crack in a glass sample is carried out by the strobo-photoelastic method to explore the propagation properties in the ultrasonic wave field under existing residual stress. Imaging results of ultrasonic wave are captured by the C-MOS camera and they were processed with integration, subtraction and normalization to enhance the contrast of the images. In the glass sample, the crack was introduced to make a distribution of static residual stresses. Residual stress was preliminary imaged by the commercial tint color strain detector to identify and to locate the distribution of stress. In the experiments for the ultrasonic wave propagation around the crack tip, wavefront was clearly visualized and its brightness was affected by the birefringence of residual stress. As the results, dynamic compressive stress by the ultrasonic wave was successfully visualized and illuminous in the static residual stress field.
\end{abstract}

Key Words : ultrasonic wave, crack, residual stress, strobe photo-elastic method

\section{Introduction}

The detection of cracks in a solid is an important issue of ultrasonic nondestructive testing. Cracks can be broadly categorized as an opened crack or a closed crack. Although an opened crack is detectable using the conventional pulse-echo method, a closed crack with a width of several nanometers may not be detected because the ultrasound transmits through the crack [1-2]. Higher harmonic detection techniques such as Contact Acoustic Nonlinearity (CAN) [3-5] have been developed to locate closed cracks or local plastic deformations in the material [6], but these methods cannot fully identify the behavior of the ultrasonic wave around the defects. Photoelastic method is the representative method to visualize the stress or ultrasonic wave propagation in the transparent material. Many previous studies [7-11], however, have not detailed with the wave propagation around the closed crack, especially tip of crack in the sample.

In this paper, the visualization of ultrasonic wave around a crack which changes from opening condition to the closed one in a glass sample is carried out by the strobo-photoelastic method to explore the propagation properties of ultrasonic wave. The effect of the residual stress in the sample around the closed crack and their tip is also described. Possibility of pilot tool as the quantitative measurement system is also explored.

\section{Sample and measurement system}

A glass sample with a crack was used to visualize the ultrasonic wave propagation in solid material, a photograph of which is shown in Figure. 1 and whose shape and dimensions are shown in Figure. 2. The longitudinal and shear ultrasonic wave velocities in the glass sample were 5500 and $2700 \mathrm{~m} / \mathrm{s}$, respectively. A rectangular piezoelectric transducer (Fuji Ceramic Co. Ltd. C-9) with a length of $30 \mathrm{~mm}$, a width of $5 \mathrm{~mm}$ and a thickness of $2 \mathrm{~mm}$ was bonded on the glass surface with silver conductive paste, where the resonant frequency of the transducer was $1.03 \mathrm{MHz}$. In this case, the wavelength was $5.5 \mathrm{~mm}$ (velocity/frequency) which makes clear observation of the wave front. The crack, which was located $25 \mathrm{~mm}$ from the top surface of the glass sample and

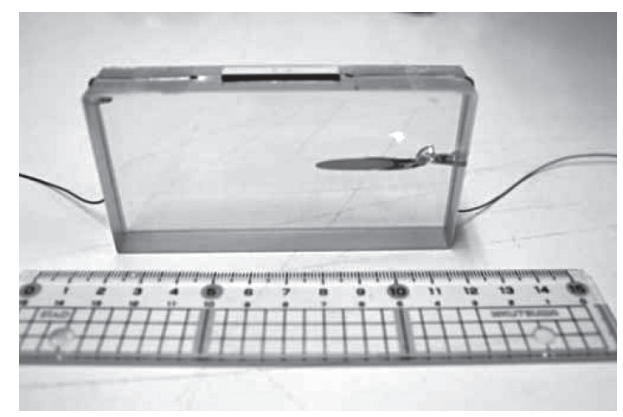

Figure. 1 Photograph of glass sample having a crack

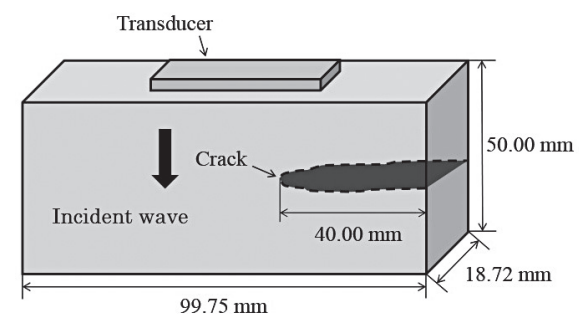

Figure. 2 Geometrical dimensions of glass sample and crack 


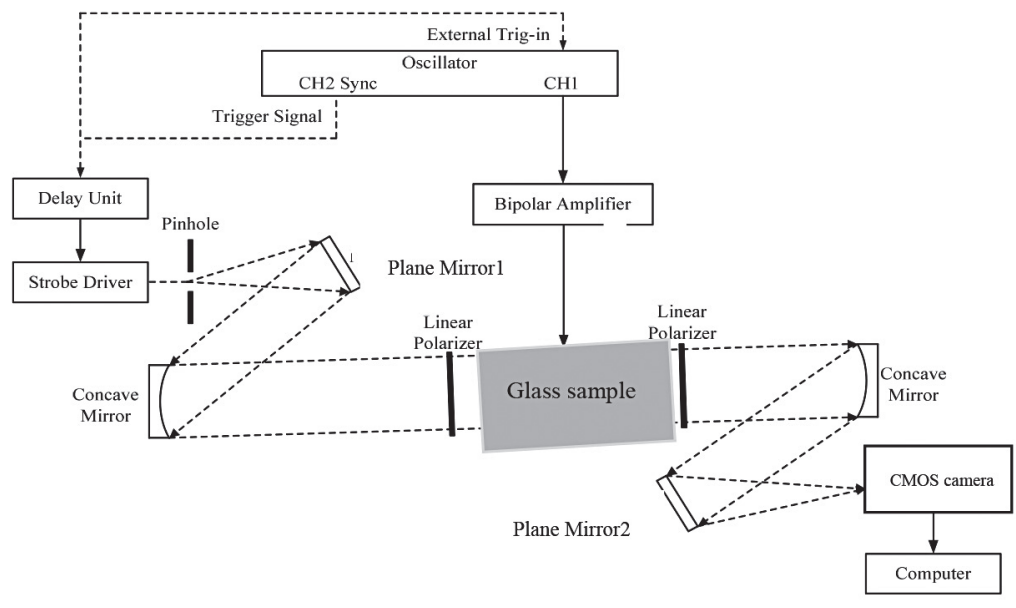

Figure. 3 Visualization system of pulsed strobo-photoelstic imaging for the crack in the glass sample

extended $40 \mathrm{~mm}$ toward the inside of the glass, was created as follows: One side of the glass was scratched to initiate the starting point of the crack, and the glass sample was then heated up to 120$140^{\circ} \mathrm{C}$ and was subsequently quenched. This procedure created a crack whose tip extended from the surface to the inside of the glass. The temperature at the creation of the crack was determined empirically, and the length of the crack could be roughly controlled by the temperature setting. The average width of the crack was estimated to be from sub-micrometer to several micrometer ranges near the surface using liquid penetrant testing method [12], though the crack tip was closed by the internal compressive stress. Figure. 3 shows the visualization system for ultrasonic wave propagation around the crack. First, a glass sample was placed between two concave mirrors, and an excitation voltage signal (CH1) from a function generator (NF Corp., WF1974) was amplified to $130 \mathrm{Vpp}$ by a bipolar amplifier (NF Corp., HA4101A) and applied to the transducer on the glass sample. Pulsed light (Sugawara Lab., NP1A) with flash time duration of $70 \mathrm{~ns}$ was expanded by passing it through a pinhole and across a mirror (Plane Mirror 1), where the repetition frequency of the flash was controlled by a function generator $(\mathrm{CH} 2)$. Collimated light propagated in the glass sample after passing through the linear polarizer and was modulated by the ultrasonic wave, after which the polarized and modulated light was received by the CMOS digital camera (Artray: ARTCAM200CMV-USB3) after passing through a second linear polarizer and being reflected by a mirror (Plane Mirror 2). Specifically, the two polarizing plates on either side of the glass sample were used to create an orthogonal Nicol state. From the constrain of the experimental set-up, the optical sample size is limited less than $90 \mathrm{~mm}$. In the experiment, the excitation voltage was designed to be a 20 -cycle, $1.03 \mathrm{MHz}$ burst sine wave with a burst interval of $10 \mathrm{~ms}$. The timing of the strobe light pulse was arbitrarily delayed using a pulse delay generator with a time resolution of $10 \mathrm{~ns}$, thereby allowing that an arbitral delay, $\Delta t$, of "frozen" ultrasonic field for an arbitral time could be obtained.

The digital sound field image data were transferred from the memory of the digital camera to a computer. Transferred data in computer memory were processed to enhance the contrast of image as illustrated in Figure. 4 (a),(b) and (c). These images were acquired by the following procedures. 1) The normal photoelastic image of ultrasonic wave propagation (a) was captured. To eliminate the fluctuations of light source, 100 pictures of image were integrated, averaged and normalized. Figure. 4 (a) is processed image. 2) The same process was applied for the background image (without sound) (b). Then, 3) processed images (a) was subtracted by image (b) to make an differential image of (c). Using these procedures, the high contrast ultrasonic sound field image shown in Figure. 4 (c) can be obtained without high voltage excitation of piezoelectric source transducer [9]. Image processing to obtain like a Figure. 4 (c) takes about 3 minutes.

\section{Experimental results}

The ultrasonic wave propagation images obtained using the photoelastic method with varying delay times, $\Delta \mathrm{t}$, of $0.5,4.0,4.5$, 5.5 and $9.0 \mu \mathrm{s}$ are shown in Figure. 5 (a-e), respectively. Ultrasonic wave propagates downward from the top. It is found that a bright part which concentrates the residual stress is clearly visualized and wave front in the residual stress zone becomes luminous.

Figure. 6 shows the sensitive tint color image [14] (Mecan imaging Co. Ltd.:MSRV-YS05Z) of glass sample, wherein the blue and pink areas correspond to the static compressive and tensile stresses, respectively. As seen in Figure. 6, residual stress is distributed into two cone-shaped areas (traced by dotted lines) around the crack. Therefore, the vertical compressive stress is distributed in two cone-shaped areas (white arrows) at a right angle to the chip and horizontal stress (black arrow) is distributed toward to the crack tip, which means that the inner residual stress is concentrated around the crack tip.

This causes the region around the crack tip to become brighter (Figures. 5(c) and (d)) owing to the increase in birefringence. Moreover, from a careful observation of the wave front, it can be seen that the velocity of sound in the compressive stress area is slightly greater than that of outside zone. Therefore, the inner compressive stress increases the velocity of sound. Although the residual stress changes in the vertical direction, stress pattern does not clearly changed. For more precise measurement, other method should be combined to our system.

As another performance of the system, it is found that the most of the sound cannot transmit through the crack, even though its width is in the sub-micrometer range, because the acoustic characteristic impedance of air $\left(408 \mathrm{Ns} / \mathrm{m}^{3}\right)$ is much lower than that of the glass $\left(12.8 \times 106 \mathrm{Ns} / \mathrm{m}^{3}\right)$ and the ultrasound is almost completely reflected by the upper crack surface. Additionally, a white mottling pattern (between the two braces) that propagates along the surfaces of 


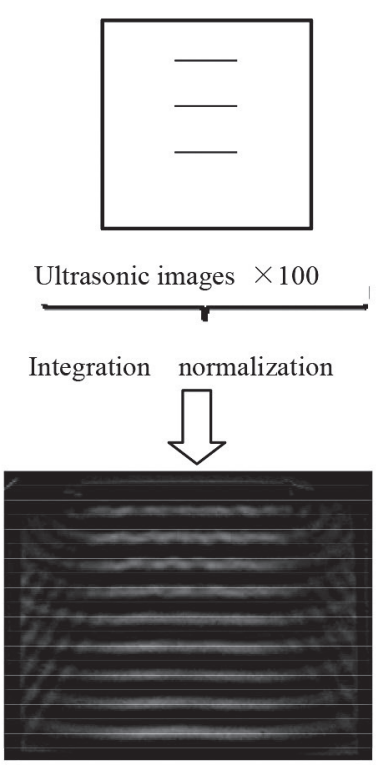

(a)

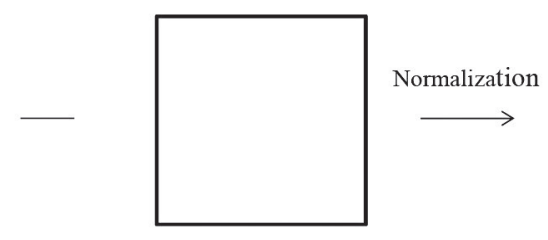

Ultrasonic images $\times 100$

Integration normalization

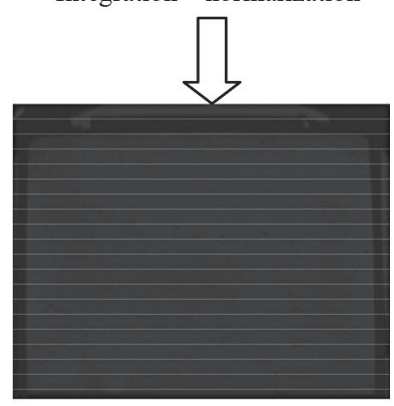

(b)

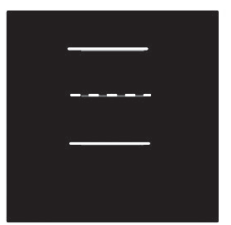

After Processing

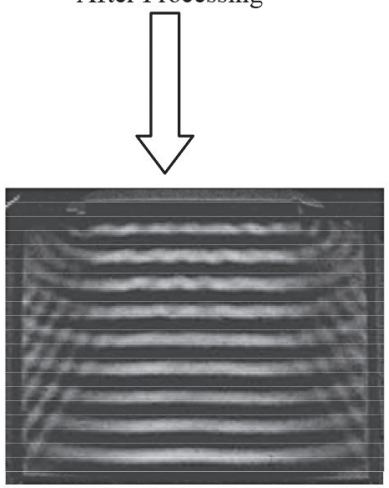

(c)

Figure. 4 Image processing method to enhance the sound field image [13].

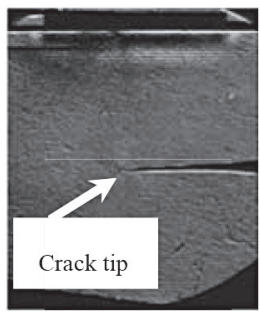

(a) $\Delta t=0.5 \mu \mathrm{s}$

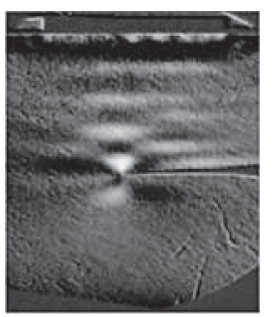

(d) $\Delta t=5.5 \mu \mathrm{s}$

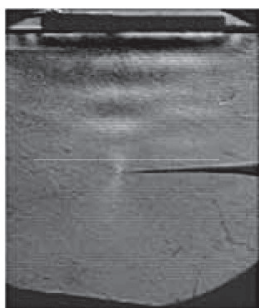

(b) $\Delta t=4.0 \mu \mathrm{s}$

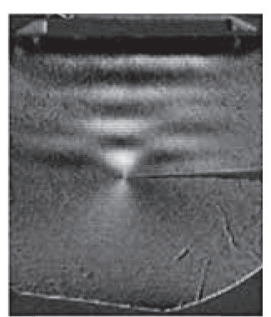

(c) $\Delta t=4.5 \mu \mathrm{s}$

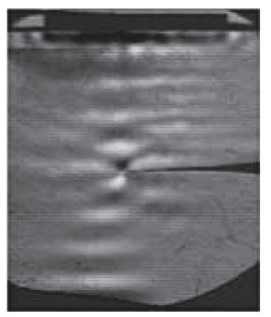

(e) $\Delta t=9.0 \mu \mathrm{s}$

Figure. 5 Photo elastic images of glass sample with a crack surrounded by residual stress.

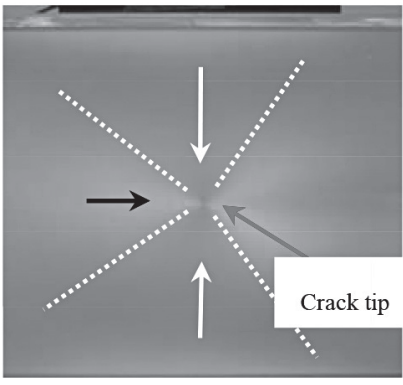

Figure. 6 Sensitive tint color image of glass sample obtained with the commercial system. Two cone type areas are traced by white dotted line. Vertical and Horizontal stress is indicated by white and black arrow, respectively.

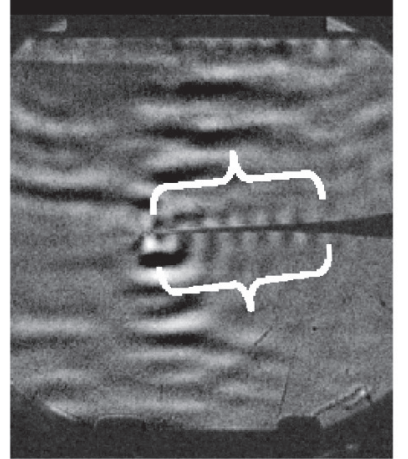

Figure. 7 Surface wave (white mottling pattern) along the crack surface obtained at 45 degrees away condition from the orthogonal Nicol condition. 
crack is observed in Figure. 7. This component is observed using the conditions wherein the light axis was shifted $45^{\circ}$ from the orthogonal Nicol state. From the propagation velocity, this wave estimated as the Rayleigh wave launched at the crack tip. Thus other component such as shear waves can be observed using the circularly polarized plates.

\section{Conclusions}

The visualization of ultrasonic wave propagation in a glass sample with a crack possessing inner stress is accomplished. To enhance the contrast of image, image processing of integration, normalization and subtraction for the photoelastic images has been executed. As the experimental results, interesting phenomena which ultrasonic wave front propagates in the residual stress zone becomes luminous. This fact reveals the regarding the ultrasonic wave propagation in the internal residual stress field around the crack tip. Additionally, it was found that other information such as shear wave or surface wave on the crack surface can also be obtained by way of taking a polarized light. Further quantitative observations of sound propagation patterns may be needed using an internal stress measurement method such as Senarmont method.

Our future work is to construct the improved system combining both the sensitive tint color and the Senarmont method to provide a quantitative measurement.

\section{References}

[1] G.L. Workman and D. Kishoni ed.: Nondestructive Testing Handbook, Vol.7 $3^{\text {rd }}$ Edition, American Society for Nondestructive Testing (2997).

[2] K. Kawashima et. al., "A nonlinear ultrasonic imaging system is developed for detecting and imaging of minute damage and defects of nm gaps in industrial materials, which are undetectable by conventional ultrasonic testing”, Vol.54, no.9 pp.509-514 (2005)(in Japanese).

[3] I. Yu. Solodov and Chin An Vu: "Nonlinear Scattering of
Acoustic Waves by Discontinuity-like Defects in Application to Crack Detection “, Acoust. Phys. 39 [1], (1993) 476.

[4] I. Yu. Solodov: "A Nonlinear NDE using contact acoustic nonlinearity”, IEEE Ultrason. Symp., 1279-1283 (1994).

[5] O. Buck, W. L. Morris and J. M. Richardson: "Acoustic harmonic generation at unbounded interfaces and fatigue cracks", Appl. Phys. Lett., 33, (1978) 371.

[6] M. Fukuda, K. Imano, H. Yamagishi and K. Sasaki : Detection of the second-harmonic of Lamb waves in fatigue magnesium plates", Acoust. Sci. \& Tech., 32 [6], (2011) 271.

[7] H. Aben, J. Anton and A. Errapart, "A Modern Photoelasticity for Residual Stress Measurement in Glass”, Strain, Vol.44, pp.40-48 (2008).

[8] E. Umezaki: "Stress Distribution Measurement Techniques Using Photoelasticity : Current Status and Future Prospects", precision Engineering, Vol79, No.7, pp.607-611 (2013) (in Japanese)

[9] T. Mihara: "Visualization of ultrasonic propagation by photoelastic image processing method", Visualization Soc. Jpn., 18 [70], 181-186 (1998). [in Japanese]

[10] K. Sekino, T. Furukawa and K. Shimizu: "Visualization of wave motion around crack by photoelastic technique", "gijyutu Rebu-“ 3, 15-19 (2007). [in Japanese]

[11] R. C. Wyatt, "Visualization of pulsed ultrasound using stroboscopic photo elasticity", Non-Distractive Testing, Dec. 354-358 (1972).

[12] Ooka ed., Nondestructive inspection (Jpn. Std. Assoc., Tokyo, 2012). [in Japanese]

[13] K. Imano: "Optical observation method for ultrasonic field using the shadowgraph introducing pulse inversion averaging”, IEICE Elex, Vol.11, No.7, pp.1-6 (2014).

[14] A. Sovalasit, G. Petrucci, and M. Scafidi, "Photoelastic Analysis of Edge Residual Stresses in Glass by the Automated Tint Plate Method", Experimental Techniques, Vol.39, pp.1118 (2005). 